\title{
Influence of the Second Bending Mode on the Response of High-Speed Bridges at Resonance
}

\author{
P. Museros and E. Alarcón
}

\begin{abstract}
This paper deals with the assessment of the contribution of the second bending mode to the dynamic behavior of simply supported railway bridges. Traditionally the contributions of modes higher than the fundamental have been considered of little importance for the computation of the magnitudes of interest to structural engineers (vertical deflections, bending moments, etc.). Starting from the dimensionless equations of motion of a simply supported beam subjected to moving loads, the key parameters governing the dynamic behavior are identified. Then, a parametric study over realistic ranges of values of those parameters is conducted, and the influence of the second mode examined in detail. The main purpose is to decide whether the second mode should be taken into account for the determination of the maximum displacement and acceleration in high-speed bridges. In addition, the reasons that cause the contribution of the second bending mode to be relevant in some situations are highlighted, particularly with regard to the computation of the maximum acceleration.
\end{abstract}

\section{Introduction}

The dynamic behavior of railway bridges constitutes a subject of research for scientists and engineers since the 19th century, but a particular growth of the interest on this topic has occurred during the last decades. The main reason for this is the extensive construction of new high-speed lines in developed countries, where the operating speed of the trains $(83.33 \mathrm{~m} / \mathrm{s}$, equal to $300 \mathrm{~km} / \mathrm{h}$, or even faster) is likely to give rise to resonance phenomena, specially in simply supported bridges.

Two different kinds of resonance phenomena are described in the technical literature related to moving loads. Since only one of them is analyzed in the present paper, an initial distinction regarding the meaning given of the term "resonance" should be made: In the rest of the paper this word will be used only to designate the rhythmic vibration of a bridge due to the passage of repeated groups of loads. The other type of resonance, which is related to the dynamic stability of beams when the speed of the moving loads reaches a certain limit (Frýba 2001), will not be investigated in this work.

When a structural engineer faces the dynamic analysis of a railway bridge, it is most likely that he will create a numerical model of the structure and, subsequently, analyze it by the mode superposition method. The minimum number of modes required for an accurate analysis, however, is an issue that creates some controversy.

The present paper deals with this important matter. The scope of the investigation is intentionally restricted to simply supported, beam-like structures such that they can be idealized as EulerBernoulli beams. It is believed that the conclusions presented herein will give enhanced understanding of the resonance behavior of railway bridges, as well as help structural engineers to decide how many modes should be included in their analyses.

\section{Purpose of Investigation}

In many developed countries of Western Europe, as well as in other parts of the world, an effort has been launched by national authorities with the purpose of updating the existing building codes for railway bridges. One of the most important reasons for this initiative was the repeated occurrence of resonant oscillations in several simply supported bridges located in the first French high-speed lines, as reported by the D-214 Committee of the European Rail Research Institute (ERRI D-214 2001).

Among the different factors that are to be considered for the design of bridges, special attention is now being paid to the verification of the serviceability limit states. As it is known, underlying these limit states are the strict requirements associated with both the passenger comfort and the security levels imposed on modern high-speed trains; as a matter of fact, in a large number of situations the serviceability requirements are the most demanding ones and become crucial for the design of railway bridges. Perhaps the most classic serviceability verification is the one concerning the vertical deflection of the deck; during recent years, in addition, the vertical acceleration of the deck has emerged as a variable that must be kept under certain limits in order to ensure 


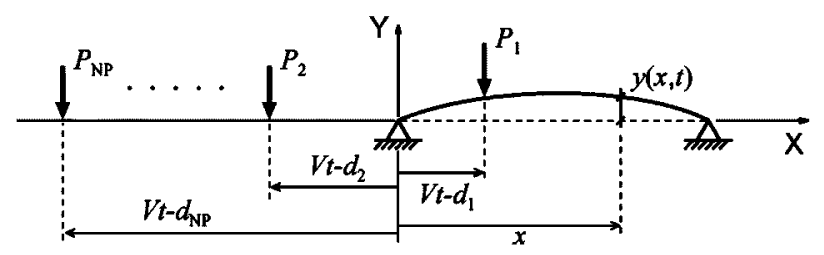

Fig. 1. Simply supported beam traversed by series (train) of concentrated loads

the stability of the track. Hereafter the attention of the paper will be focused on these two factors due to their paramount importance

Yang et al. indicate that the contribution of the fundamental bending mode suffices for an accurate computation of the dynamic deflection of a simply supported beam (Yang et al. 1997); the classic text (Biggs 1964) is cited in this reference as the primary source. Similarly, Li and Su (Li and Su 1999) cite the text (Smith 1988) to affirm that the bending moment, as well as the deflection, can be computed correctly neglecting the contribution of bending modes higher than the first one.

However, the ERRI D-214 committee proposes that the second mode should also be included if the associated natural frequency is lower than $30 \mathrm{~Hz}$ (ERRI D-214 2001). This recommendation is based on the fact that accelerations up to that frequency could negatively influence ballast stability.

The purpose of the paper is to further investigate the necessity of including the second bending mode in the dynamic analysis of railway bridges, not only for the evaluation of the maximum acceleration, but also for the computation of the maximum displacement. The reason that the authors have found for pursuing this question is the possibility that the second bending mode of a simply supported bridge could be excited at resonance during the passage of a high-speed train. The present investigation will show that indeed such a situation could take place, which creates an uncertainty regarding the influence that the second mode could have on the assessment of the maximum displacements of highspeed bridges.

\section{Equation of Motion and Similarity Formulas}

\section{Mathematical Model}

The approach adopted in the present investigation is based on the so-called similarity formulas. These formulas have been used by the ERRI (ERRI 2001), and were also exploited by the authors in several previous works (Museros et al. 1999; 2002; Museros and Alarcón 2002).

The validity of the similarity formulas can be proved on the basis of the equation of motion of a simply supported beam of constant mass per unit length and constant cross-section properties, traversed by a series of concentrated loads (see Fig. 1). This equation has been presented by a number of authors (Klasztorny and Langer 1990; Olsson 1991; Frýba 1999; Li and Su 1999 among others). The following dimensionless form has been used previously by the authors (Museros 2002); the derivation is also included in the Appendix:

$$
\begin{aligned}
\frac{1}{8 \pi^{2}} \xi_{n}^{\prime \prime}(\tau)+\frac{n^{2} \zeta_{n}}{2 \pi} \xi_{n}^{\prime}(\tau)+\frac{n^{4}}{2} \xi_{n}(\tau) & \\
= & \frac{-1}{\omega_{0}^{2} \cdot m L} \sum_{k=1}^{N P}\left(H\left(\tau-\frac{d_{k}}{\alpha L}\right)-H\left(\tau-\frac{d_{k}}{\alpha L}-\frac{1}{\alpha}\right)\right) P_{k} \\
& \times \sin \left(n \pi\left(\alpha \tau-\frac{d_{k}}{L}\right)\right)
\end{aligned}
$$

In Eq. (1) the following notation is used: $\tau=$ dimensionless time $\tau=t / T$, where $t=$ real time and $T$ symbolizes the period of the first bending mode (i.e., the fundamental period); primes denote derivation with respect to dimensionless time; $\omega_{0}=2 \pi / T=$ fundamental frequency; $n$ indicates the number of the mode; and $\xi_{n}(\tau)=$ dynamic amplitude of the $n$th mode at time $\tau$; $\zeta_{n}$ represents the damping ratio of the $n$th mode; $L$ and $m=$ length and constant mass per unit length of the bridge, respectively; $P_{k}$ and $d_{k}$ stand for the value of the $k$ th concentrated load and its initial distance from the beginning of the bridge (usually $d_{1}$ is given a zero value); $N P=$ total number of concentrated loads of the train; and finally $\alpha=$ dimensionless speed parameter representing the fraction of the length $L$ traveled by the train during one fundamental period: $\alpha=V T / L$, where $V=$ speed of the train.

Also, in Eq. (1) $H\left(\tau-\tau_{0}\right)$ denotes the Heaviside unit function

$$
H\left(\tau-\tau_{0}\right)=\begin{array}{ll}
0 & \tau<\tau_{0} \\
1 & \tau \geqslant \tau_{0}
\end{array}
$$

The deformed shape of the beam $y(x, t)$ is obtained to the desired degree of accuracy by superposition of the required modal contributions, and the modes of vibration are given by the usual family of sines: $\phi_{n}(x)=\sin (n \pi x / L)$. The abscissa along the span of the bridge is represented by $x$. After conversion from dimensionless time to real time, the expression of the deformed shape is as follows:

$$
y(x, t)=\sum_{n=1}^{N} \xi_{n}(t) \phi_{n}(x)=\sum_{n=1}^{N} \xi_{n}(t) \sin \frac{n \pi x}{L}
$$

The selection of such a simple mathematical model for the analysis of railway bridges deliberately neglects the influence of several factors. The main reason for adopting this approach is that high-speed bridges, particularly the ones located in international lines, must be designed on the basis of conservative hypotheses because different kinds of trains, the ones existing nowadays but also new ones, are likely to circulate the bridges during its life. Therefore, the analysis of the structure should not be carried out considering the particular characteristics of the vehicles (e.g., suspension system, unsprung wheel masses, etc.). This is of great importance with regard to train-bridge interaction, which the authors have investigated in previous works showing that it has beneficial effects on the bridge response (Museros and Alarcón 2002; Museros et al. 2002), but verifying simultaneously that these beneficial effects are strongly dependent on the primary suspension system of the vehicle. Particularly, from the numerous real cases considered during the investigations presented in $\mathrm{Mu}-$ seros and Alarcón 2002, the authors found that, due to the trainbridge interaction effects, the response of the bridge at resonance was almost always reduced and sometimes very slightly increased. Therefore, it was felt that neglecting the influence of the vehicle sprung masses and suspension system was a conservative approach convenient for the analysis of high-speed bridges. This point of view is also present in Eurocode 1 (CEN 2002a), which suggests that the dynamic analyses should be based on moving 
loads models. If desired, the beneficial effect of train-bridge interaction can be taken into account by means of a suitable increment of the structural damping ratio. Nevertheless, a different, more sophisticated approach based on vehicle-bridge interaction models, could be more adequate if the characteristics of all vehicles allowed to circulate over a bridge were reliably known a priori.

Another factor that is neglected by the mathematical model adopted in this paper is the load distribution due to the presence of the sleepers and the ballast layer. This effect tends also to reduce the response of the bridge, but the authors (Museros 2002; Museros et al. 2002) and the ERRI (ERRI D-214 2001) have shown that it is significant to some extent only for the shortest spans ( $L<15 \mathrm{~m}$, approximately). Consequently, ignoring such effect has no consequence for the vast majority of simply supported bridges, while implies a slightly conservative approach in the analysis of the shortest ones.

Also, according to Yang et al., the Coriolis effect created by the passage of the wheels over the deflected bridge can be neglected as a result of the great stiffness of the structures used in high-speed lines (Yang et al. 1997).

Finally, the track irregularities can excite the vibration of some modes depending on their position along the bridge. In the present investigation this effect will not be taken into account and attention will be focussed on the rhythmic vibrations induced by the traveling loads, which are the subject of main concern when resonant behavior occurs. The increase of the structural response due to the presence of track irregularities can be accounted for approximately following the recommendations of Eurocode 1 (CEN 2002a)

\section{Similarity Formulas and Fundamental Parameters}

In the present section two systems, each of them consisting of a simply supported bridge and a train of $N P$ concentrated loads crossing over it, will be considered. Furthermore, it will be assumed that the values of the loads $P_{k}$ are equal in both trains. If the values of some particular parameters shown in Eq. (1) are the same in both systems and, besides, dimensionless time $\tau$ is used as the independent variable, then the time-histories of every pair of corresponding modal amplitudes $\xi_{n}$ turn out to be proportional. These parameters are the following: modal damping ratios $\zeta_{n}$, dimensionless speed $\alpha$, and dimensionless ratios between the load distances $d_{k}$ and the bridge $\operatorname{span} L$ (i.e., $d_{k} / L$ ratios).

In such conditions it is apparent from Eq. (1) that the values of all terms are the same for both train-bridge systems except for $\omega_{0}^{2} \cdot m L$ on the right-hand side (generally this term will vary from system to system). Therefore the $n$th modal amplitude at time $\tau$, $\xi_{n}(\tau)$, will be inversely proportional to $\omega_{0}^{2} \cdot m L$.

In conclusion, if the values of the parameters mentioned above $\left(\zeta_{n}, \alpha\right.$, and $\left.d_{k} / L\right)$ are the same in two train-bridge systems, these systems are said to be similar, and the following relation between their responses can be formulated:

$$
y_{1}\left(x_{1}, t_{1}\right)=\frac{m_{2} L_{2}}{m_{1} L_{1}}\left(\frac{n_{02}}{n_{01}}\right)^{2} y_{2}\left(x_{2}, t_{2}\right) \quad \frac{t_{1}}{T_{1}}=\frac{t_{2}}{T_{2}}=\tau \quad \frac{x_{1}}{L_{1}}=\frac{x_{2}}{L_{2}}
$$

where $n_{01}$ and $n_{02}$ symbolize the fundamental frequencies of both bridges in Hertz. The rest of the variables retain their meaning, and subscripts 1 and 2 are used to denote whether they are related to the first or the second train-bridge system. The responses $y_{1}\left(x_{1}, t_{1}\right)$ and $y_{2}\left(x_{2}, t_{2}\right)$ are computed according to Eq. (3). If Eq. (4a) is differentiated twice with respect to dimensionless time, a relation between the vertical accelerations $a(x, t)$ is obtained

$$
a_{1}\left(x_{1}, t_{1}\right)=\frac{m_{2} L_{2}}{m_{1} L_{1}} a_{2}\left(x_{2}, t_{2}\right) \quad \frac{t_{1}}{T_{1}}=\frac{t_{2}}{T_{2}}=\tau \quad \frac{x_{1}}{L_{1}}=\frac{x_{2}}{L_{2}}
$$

In Eq. (4b) the vertical accelerations must be computed by differentiation with respect to real time

$$
a_{j}=\frac{d^{2} y_{j}}{d t_{j}^{2}}=\frac{d^{2} y_{j}}{d \tau^{2}} \cdot\left(\frac{d \tau}{d t_{j}}\right)^{2}=\frac{d^{2} y_{j}}{d \tau^{2}} \cdot n_{0 j}^{2} \quad j=1,2
$$

By virtue of Eqs. (4a) and (4b), the maximum responses of two similar train-bridge systems are related by the expressions

$$
\begin{gathered}
\max \left\{y_{1}\left(x_{1}\right)\right\}=\frac{m_{2} L_{2}}{m_{1} L_{1}}\left(\frac{n_{02}}{n_{01}}\right)^{2} \max \left\{y_{2}\left(x_{2}\right)\right\} \\
\max \left\{a_{1}\left(x_{1}\right)\right\}=\frac{x_{1}}{L_{1}}=\frac{x_{2}}{m_{1}} \\
m_{1} L_{1} \\
\max \left\{a_{2}\left(x_{2}\right)\right\}
\end{gathered}
$$

Eqs. (4a), (4b), and (6) form the theoretical basis of this investigation and will be referred to as the similarity formulas. More precisely, this research work is based on the fact that, as the above formulas reveal, if resonance condition takes place in a bridge traversed by a train of moving loads, the same will happen in all similar train-bridge systems.

Finally, one restriction of the similarity formulas should be mentioned. Since the distances between the loads of real trains have fixed values, the application of these formulas is restricted in practice to bridges of equal length (otherwise the values of the ratios $d_{k} / L$ would not be the same). In the next sections this limitation will be overcome by means of a parametric analysis over a realistic range of span lengths.

\section{Hypotheses for Parametric Study}

As mentioned previously, the purpose of this investigation is to analyze the influence of the second bending mode on the dynamic behavior of railway bridges. This will be accomplished by means of a parametric study of which the hypotheses are described below.

\section{Structural Damping}

The selection of appropriate damping ratios for the different modes of vibration is of great importance in structural dynamics. Following the recommendations of the ERRI (ERRI D-214 2001), $1 \%$ damping has been selected since it represents a typical value for many prestressed concrete bridges. Values higher than $1 \%$ are expected in short span bridges but, since the span length is not a primary variable in this study (this will be shown in the following sections), it is not straightforward to associate different lengths with different damping ratios, as it is customary. On the contrary, one of the primary variables is the dimensionless ratio between the span length $L$ and the characteristic distance $d$; accordingly, it proves more convenient to use a single value of the damping ratio in order to obtain more consistent results.

The recommendations of ERRI D-214 follow from a number of experimental results obtained in field tests. Nevertheless, the results furnished by most of the tests carried out in practice are related to the fundamental bending and torsional modes only. 
Therefore, there is little information available regarding damping ratios of higher modes. In this study it is assumed that the damping ratios of the first and second mode are the same: $\zeta=1 \%$; according to the ERRI and Frýba it is expected that higher modes exhibit higher damping ratios and, consequently, this should be a conservative hypothesis (Frýba 1996; ERRI D-214 2001).

\section{Type of Train}

In normal operation (i.e., excluding seismic or abnormal excitation), the most demanding situation for a simply supported bridge is the development of resonant response during the passage of a train. Therefore, a train consisting of equally spaced, constantvalued loads, capable of exciting a bridge at resonance, has been selected for this parametric study. After consideration of the number of repeated groups of loads of actual high-speed trains (usually equal to or very close to the number of passenger coaches), the number of loads chosen for the train is $N P=15$, each of them having a value of $1 \mathrm{~N}$. The distance between every two consecutive loads $d$ has been adjusted so as to reproduce a variation over a range of one of the fundamental parameters, as will be explained later.

This simple type of train does not match exactly the patterns of loads of most real trains, but it is suitable for exciting bridges at resonance and, therefore, is adequate for capturing the essential features of the dynamic behavior of railway bridges in such conditions. Besides, as will be explained in the next section, this type of train with concentrated loads represents a conservative approach compared to real trains.

\section{Fundamental Parameters}

Having fixed the type of train and the damping ratios for the first and second modes, only two independent parameters characterize the equation of motion. These are the dimensionless speed $\alpha$ and the dimensionless ratios $d_{k} / L$. Since the train consists of equally spaced loads, the distances $d_{k}$ can be expressed simply as

$$
d_{k}=(k-1) d \quad k=1,2, \ldots 15
$$

where $d$ symbolizes the distance between two consecutive loads, as mentioned before. Therefore, $d_{k} / L=(k-1) d / L$, and the differential equation of motion depends solely on the values of $\alpha$ and $d / L$. In what follows the inverse relation $L / d$ will be used, but this is simply a matter of personal preference.

The parametric study, in conclusion, can be carried out provided that suitable ranges of values are defined for $\alpha$ and $L / d$. The results of this study will enable a comparison between the relative influence of the first and second bending modes. The next two sections are devoted to the determination of realistic ranges of values for $L / d$ and $\alpha$.

\section{Range of Values of $L / d$}

A realistic range of values of $L / d$ can be defined by assuming maximum and minimum values of both variables.

With regard to the length of the bridge, the usual values in simply supported structures for high-speed lines are greater than $10 \mathrm{~m}$ and smaller than $50 \mathrm{~m}$. These can be considered de facto limits in common practice.

The determination of a range of values for the characteristic distance $d$ requires more sophisticated considerations. Modern high-speed European trains can be classified in three groups (see Fig. 2). Conventional trains adhere to the classic configuration where the weight of each passenger ear is supported by two bo-

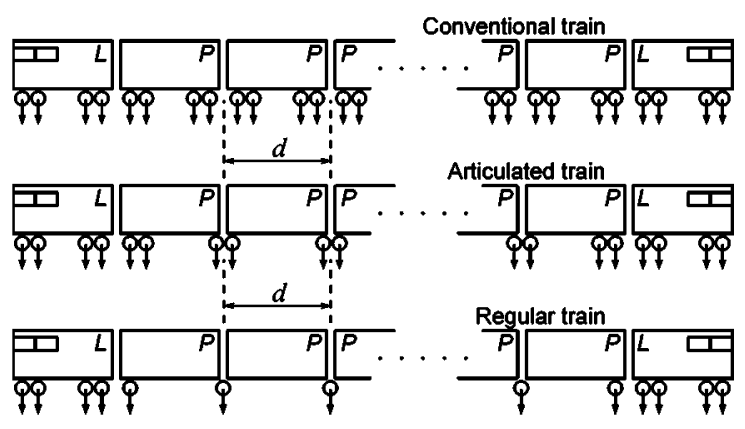

Fig. 2. Different types of modern high-speed trains: $L=$ locomotive; $P=$ passenger car

gies, a front one and a rear one, each of them carrying two axles; examples of conventional trains are the ICE, ETR-Y-500 (also known as Pendolino) and Virgin Express. The pattern of loads of articulated trains is derived from the former by merging every pair consisting of a rear bogie and the front bogie of the next car into a single bogie (which is shared by both cars); among the articulated trains one can mention the TGV, Thalys, Eurostar, and AVE. Finally, regular trains differ from articulated trains essentially in that the two axles of every bogie are merged into one; an example of such trains is the Spanish Talgo. The locomotives are excluded from this classification since, regardless of the type of train, they usually consist of a car-body supported in front and rear bogies, each of them incorporating two axles.

Resonance is produced by the periodic repetition of isolated loads or groups of loads circulating along the bridge. Thus, it can be deduced from Fig. 2 that the most important distance related to the appearance of resonance must be the length of the passenger cars. Although there is also a possibility of resonance due to the consecutive passage of the two bogies of a locomotive, and also due to other combinations of intermediate loads, these are always resonance situations with a very limited duration, and their importance is usually small compared to the main resonance created by the rhythmic passage of the cars. This is a fact that the authors have confirmed during the development of numerous studies related to the $\mathrm{PhD}$ thesis (Museros 2002). Therefore, in the present study the length of the passenger cars is considered to be the main characteristic distance between repeated groups of loads and, consequently, will be associated with the distance $d$ between consecutive loads of the theoretical train defined previously.

Identifying the car length and the distance $d$ is truthful regarding regular trains, but on the other hand introduces an approximation in the modeling of conventional and articulated trains. It is well known that in simply supported beams the static effects of a concentrated load diminish if the load is distributed over a certain distance; the authors have shown in previous works (Museros et al. 2002) that the same happens when the loads travel over the beam at a constant speed. Therefore it is expected that, if the two loads of a bogie of an articulated train, or the four loads corresponding to the rear and front bogies of two consecutive cars of a conventional train are merged into a single load, the dynamic effects during the passage of the train will be increased. This is a fact that has also been confirmed by numerical simulations carried out during the development of the present investigation.

Hence, it turns out that analyzing the resonant effects induced by conventional and articulated trains by means of theoretical trains of equidistant loads is a conservative approach, providing that the length of the passenger cars is identified with the characteristic distance $d$ and the corresponding loads are merged into 
Table 1. Minimum and Maximum Span Length as Function of $L / d$, Dimensionless Speeds $\alpha_{2, i}$, and Maximum Values of $\alpha$ and $\Lambda$ in High-Speed Range

\begin{tabular}{|c|c|c|c|c|c|c|c|c|}
\hline$L / d$ & $L_{\min }(\mathrm{m})$ & $L_{\max }(\mathrm{m})$ & $\alpha_{\max }$ & $\alpha_{2,1}$ & $\alpha_{2,2}$ & $\alpha_{2,3}$ & $\alpha_{2,4}$ & $\Lambda_{\max }$ \\
\hline 0.30 & 3.9 & 7.9 & 1.46 & 13.33 & 6.67 & 4.44 & 3.33 & 0.44 \\
\hline 0.50 & 6.6 & 13.2 & 1.46 & 8.00 & 4.00 & 2.67 & 2.00 & 0.73 \\
\hline 0.75 & 9.9 & 19.8 & 1.46 & 5.33 & 2.67 & 1.78 & 1.33 & 1.10 \\
\hline 1.00 & 13.1 & 26.4 & 1.46 & 4.00 & 2.00 & 1.33 & 1.00 & 1.46 \\
\hline 1.25 & 16.4 & 33.0 & 1.46 & 3.20 & 1.60 & 1.07 & 0.80 & 1.83 \\
\hline 1.50 & 19.7 & 39.6 & 1.46 & 2.67 & 1.33 & 0.89 & 0.67 & 2.19 \\
\hline 1.75 & 23.0 & 46.2 & 1.38 & 2.29 & 1.14 & 0.76 & 0.57 & 2.42 \\
\hline 2.00 & 26.3 & 52.8 & 1.31 & 2.00 & 1.00 & 0.67 & 0.50 & 2.61 \\
\hline 2.25 & 29.6 & 59.4 & 1.25 & 1.78 & 0.89 & 0.59 & 0.44 & 2.80 \\
\hline 2.50 & 32.9 & 66.0 & 1.19 & 1.60 & 0.80 & 0.53 & 0.40 & 2.98 \\
\hline 2.75 & 36.1 & 72.6 & 1.15 & 1.45 & 0.73 & 0.48 & 0.36 & 3.16 \\
\hline 3.00 & 39.4 & 79.2 & 1.11 & 1.33 & 0.67 & 0.44 & 0.33 & 3.32 \\
\hline 3.25 & 42.7 & 85.8 & 1.07 & 1.23 & 0.62 & 0.41 & 0.31 & 3.49 \\
\hline 3.50 & 46.0 & 92.4 & 1.04 & 1.14 & 0.57 & 0.38 & 0.29 & 3.64 \\
\hline 3.75 & 49.3 & 99.0 & 1.01 & 1.07 & 0.53 & 0.36 & 0.27 & 3.79 \\
\hline 4.00 & 52.6 & 105.6 & 0.99 & 1.00 & 0.50 & 0.33 & 0.25 & 3.94 \\
\hline
\end{tabular}

one. Conversely, in out-of-resonance conditions the heavier axles of the locomotives are most often predominant, and thus the simplified model used in this investigation would not be an adequate representation of the actions caused by real trains.

There is the possibility that the presence of intermediate or rear locomotives modifies the amplitude of the resonant vibration. This effect can be either advantageous or disadvantageous, and requires specific calculations for each type of train. Nevertheless, in the present paper this effect will not be investigated and attention will be focused on the resonant behavior of railway bridges generated by the rhythmic passage of loads.

Bearing these simplifications in mind, maximum and minimum values of $d$ can be easily established: The shortest coaches are those of the Talgo train, where $d=13.14 \mathrm{~m}$. Conversely, the longest ones correspond to the ICE, with $d=26.4 \mathrm{~m}$. Accordingly, the resulting range of values for the $L / d$ ratio is

$$
\frac{10}{26.4} \leqslant \frac{L}{d} \leqslant \frac{50}{13.14} \quad \Rightarrow \quad 0.38 \leqslant \frac{L}{d} \leqslant 3.8
$$

For convenience, this initial range has been expanded to a final interval $0.3 \leqslant L / d \leqslant 4$, and 16 discrete numerical values have been selected in order to cover it adequately. These numerical values are shown in Table 1 along with the corresponding values of the dimensionless speed parameter $\alpha$.

\section{Range of Values of $\alpha$}

The estimation of a range of values for $\alpha$ is somewhat more involved than it was for the $L / d$ ratio.

According to the purpose of the present investigation, the speed range of the parametric analysis must be selected so as to embrace the most important resonances of the first and second modes. The $i$ th resonance of the $n$th mode is defined as the situation in which the $n$th mode completes $i$ cycles of oscillation between the passage of two consecutive loads. The corresponding speed is denoted as $V_{n, i}$, and can be computed as follows:

$$
V_{n, i}=\frac{1}{T_{n}} \cdot \frac{d}{i}=\frac{n^{2}}{T} \cdot \frac{d}{i} \quad n=1,2 \quad i=1,2,3,4
$$

In Eq. (9) the number of modes is intentionally limited to two, and only the first four resonance situations of each mode are considered. The dynamic amplification associated with a number of cycles greater than four (i.e., $i \geqslant 5$ ) is of little importance and need not be considered here. Also, in Eq. (9) $T_{n}$ stands for the period of the $n$th mode, while $T$ stands for the period of the fundamental mode $\left(T=T_{1}\right)$.

The dimensionless speed associated to each resonance situation is, according to the definition of $\alpha$

$$
\alpha_{n, i}=\frac{V_{n, i} T}{L}=\frac{n^{2}}{i} \cdot \frac{d}{L} \quad n=1,2 \quad i=1,2,3,4
$$

Whether the eight values of $\alpha$ that one can obtain from Eq. (10) are attainable for modern high-speed trains is a question that requires further investigation. An answer can be obtained if the band in Fig. 3, included in the monograph (Frýba 1996), is assumed as the most usual range of pairs \{span length, fundamental frequency $\}$ in railway bridges. Relying on this hypothesis a maximum value of $\alpha$ for each $L / d$ can be estimated. This is accomplished as follows.

First, realistic minimum and maximum values of span length are computed for each $L / d$ ratio. This is a straightforward calculation considering the realistic limits assumed for $d$ in the previous section

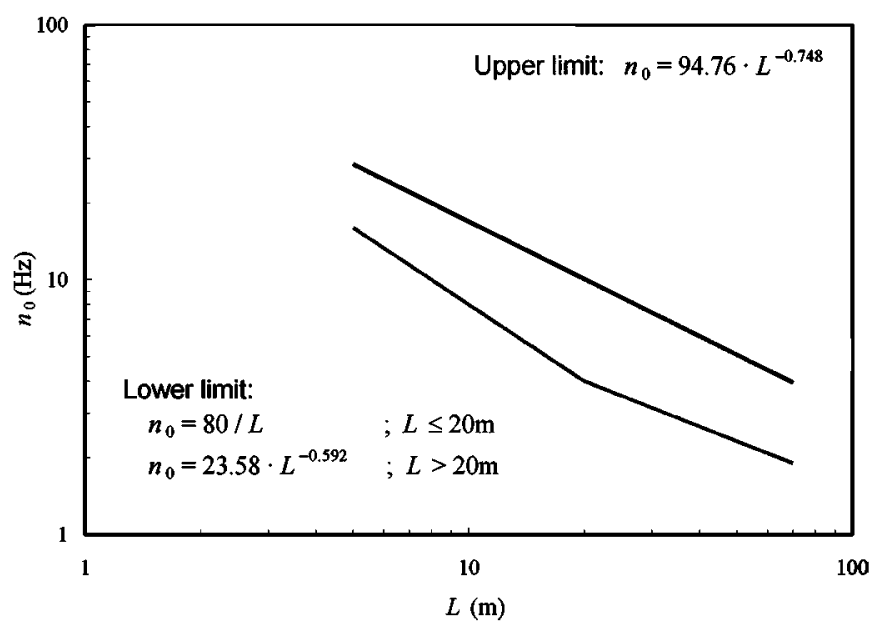

Fig. 3. Assumed range of usual spans and frequencies 


$$
L_{\min }=13.14 \cdot\left[\frac{L}{d}\right] \quad L_{\max }=26.4 \cdot\left[\frac{L}{d}\right]
$$

Eqs. (11) define an interval $\left[L_{\min }, L_{\max }\right]$ in the horizontal axis of Fig. 3. The maximum dimensionless speed in that interval is obtained when $V$ is maximum and the product $\left(n_{0} L\right)$ is minimum. From the mathematical expressions of the upper and lower limit of the band in Fig. 3, it can be shown that, within an interval of spans $\left[L_{\min }, L_{\max }\right]$, the minimum value of $\left(n_{0} L\right)$ corresponds to the point defined by the minimum length $L_{\min }$ lying on the lower limit of the band. In other words, if $L_{\min } \leqslant L \leqslant L_{\max }$, then the minimum value of $\left(n_{0} L\right)$ is

$$
\begin{gathered}
\left(n_{0} L\right)_{\min }=\left(\frac{80}{L_{\min }}\right) \cdot L_{\min }=80 \quad L_{\min } \leqslant 20 \mathrm{~m} \\
\left(n_{0} L\right)_{\min }=\left(23.58 \cdot L_{\mathrm{min}}^{-0.592}\right) \cdot L_{\min } \quad L_{\min }>20 \mathrm{~m}
\end{gathered}
$$

As a result, if a maximum speed at the site of $97.22 \mathrm{~m} / \mathrm{s}(350 \mathrm{~km} / \mathrm{h})$ is considered and the maximum design speed is computed according to the recommendations of Eurocode 1 (CEN 2002a), which leads to $V_{\max }=1.2 \times 97.22$ $=116.67 \mathrm{~m} / \mathrm{s}(420 \mathrm{~km} / \mathrm{h})$, the steps required for computing the maximum dimensionless speed for each $L / d$ ratio are as follows:

Fix a value of $L / d \rightarrow$ Compute $L_{\min }$ and $L_{\max }$ from Eqs. (11) $\rightarrow$ Compute $\left(n_{0} L\right)_{\min }$ from Eqs. (12) $\rightarrow$ Determine $\alpha_{\max }$ $=V_{\max } /\left(n_{0} L\right)_{\min }$

Table 1 shows the values of the $L / d$ ratio selected for the analysis, along with the corresponding values of $L_{\min }$ and $L_{\max }$ [obtained from Eqs. (11)], $\alpha_{\max }, \alpha_{2, i}$ (resonance situations of the second mode), and $\Lambda_{\max }$. A bold italicized entry in Table 1 indicates that, for that particular value of $L / d, \alpha_{2, i}<\alpha_{\max }$ and therefore the associated resonance of the second mode takes place at speed higher than $V_{\max } . \Lambda$ is the normalized wavelength, a useful parameter that represents the ratio of the distance traveled by the train during one fundamental period $(V T)$ and the characteristic distance between axle loads $(d)$

$$
\Lambda=\frac{V T}{d}=\alpha \frac{L}{d} \Rightarrow \Lambda_{n, i}=\alpha_{n, i} \frac{L}{d}=n^{2} / i \quad n=1,2 \quad i=1,2,3,4
$$

The values of $\Lambda$ at resonance $\left(\Lambda_{n, i}\right)$ depend only on the mode number $(n)$ and resonance number $(i)$. The first four resonances of the second mode correspond to $\Lambda=4,2,4 / 3$, and 1 , respectively. As for the resonances of the first mode, the associated values of $\Lambda$ are $1,1 / 2,1 / 3$, and $1 / 4$. Comparing these values with $\Lambda_{\max }$ $=\alpha_{\max } \cdot(L / d)$ is an alternative way of determining which resonance situations can be attained at speed lower than $V_{\max }$.

In the next sections, $\Lambda$ will be used as abscissa in several graphics displaying results of the analysis. Furthermore, for each value of $L / d$ in Table 1, 1,000 different values of speed $V$ have been selected for the analysis; these values have been equally distributed between $0.9 V_{1,4}$ and $1.1 V_{2,1}$ in order to include the first four resonances of the first and second mode. Accordingly, the values of $\Lambda$ in the horizontal axis of the graphics mentioned above will range from $0.9 \Lambda_{1,4}=0.225$ to $1.1 \Lambda_{2,1}=4.4$. The reason for using such a large number of different speeds was the need for computing the maximum response at resonance accurately, which in some cases proved rather tedious from a computational point of view due to the presence of very sharp resonance peaks. Eventually, this caused the selection of a very small interval in the speed range.
Finally, in Table 1 it should be also noted that, since the initial range specified for $L / d$ [see Eq. (8)] was expanded to $0.3 \leqslant L / d$ $\leqslant 4.0$, the span lengths associated with the extreme values are, respectively, shorter than $10 \mathrm{~m}$ and longer than $50 \mathrm{~m}$.

\section{Selection of Several Representative Sections for Evaluation of Response}

If the maximum response of the bridge is to be obtained, several values of the abscissa $x$ must be selected in order to ensure that the overall maximum is computed with accuracy. To this end, 21 different values of $x$ have been selected. These values are equally spaced between two extreme sections corresponding to $x / L=\frac{1}{4}$ (at the first quarter of span) and $x / L=\frac{3}{4}$ (third quarter of span), i.e., every two consecutive values of $x$ are separated by $\Delta(x / L)$ $=0.025$. Regarding the verification of the serviceability limit states defined in Annex A2 of the basic Eurocode (CEN 2002b), the signs of the maximum displacement and acceleration are not of concern (i.e., absolute values are to be computed); as a consequence, the maximum response will surely occur between the two mentioned sections.

\section{Characteristics of Bridge}

Because the response of similar train-bridge systems is proportional, unitary values have been selected for the main characteristics of the bridge, i.e., the length of the bridge has been taken equal to $1 \mathrm{~m}$, its mass per unit length equal to $1 \mathrm{~kg} / \mathrm{m}$, and the bending stiffness has been tuned so as to produce a fundamental frequency $n_{0}=1 \mathrm{~Hz}$.

\section{Distance Between Consecutive Loads (d)}

Since the length of the bridge is equal to $1 \mathrm{~m}$, as mentioned in "Characteristics of the Bridge," the 16 different $L / d$ ratios listed in Table 1 are obtained by means of an appropriate modification of the distance $d$ between consecutive loads. Thus, 16 different trains have been used, each of them associated with one of the following values of $d: 1 / 0.3,1 / 0.5,1 / 0.75,1 / 1,1 / 1.25, \ldots, 1 / 4$.

\section{Results}

In this section the results of the analysis are presented. First, the influence of the second mode on the maximum response of the bridge is analyzed. Afterwards, a brief discussion regarding the conditions for the cancellation of resonance is included. Presenting plots of the results for all values of $L / d$ ratio included in Table 1 requires an amount of space that would exceed the reasonable size of a paper. Therefore, figures of the results will be shown only for the most relevant cases.

\section{Influence of Second Bending Mode on Response of Bridge}

Since the investigation presented in this paper is based on a numerical analysis over a range of variation of several parameters, the conclusions that can be extracted, if affirmative, cannot be guaranteed to hold for values of such parameters different than the ones considered herein. Therefore, the approach taken is to establish whether certain statements are not valid in some particular cases.

As explained in previous sections, the bridge response has 
been computed for two different quantities, each of them related to a different serviceability limit state. These quantities are the vertical displacement and vertical acceleration of the deck. Sixteen values of the $L / d$ ratio have been considered, and the maximum absolute values (i.e., regardless of sign) of both response variables have been computed for 21 different locations along the span of the bridge; the analysis has been performed for 1,000 different values of the speed, equally spaced between $0.9 V_{1,4}$ and $1.1 V_{2,1}$.

Specifically, the purpose of the analysis is to give an answer to three different questions. Considering that the maximum design speed is $V_{\max }=116.67 \mathrm{~m} / \mathrm{s}$, and therefore the values of $\Lambda$ $=V T / d$ must be less or equal than $\Lambda_{\max }$ (see Table 1), the questions to be answered for each value of $L / d$ ratio are as follows:

1. Is the maximum response obtained at mid-span?

2. In case that the answer to question (1) were negative, is the maximum response obtained at the first quarter of span or at three quarters of span?

3. Regardless of the answers to questions (1) and (2), are the maximum values of the response computed at first and third quarters of span identical?

If the answer to question (1) is negative in a particular case, the influence of the second bending mode in that case cannot be ignored.

With respect to question (2), if the maximum response is not obtained at mid-span, this is obviously due to the contribution of the second mode. Since the maximum displacement and acceleration associated with this contribution are obtained at $x / L=\frac{1}{4}$ and $x / L=\frac{3}{4}$, it is of interest to establish whether the overall maximum (sum of the effects of first and second mode) is also obtained at those sections. If this were not so, the computation of the response at intermediate locations (such as $x / L=0.3,0.4$, etc.) would reveal as a crucial part of the analysis.

Finally, with question number (3) it is investigated whether there are cases such that the maximum response at the section located at the first quarter of span is significantly different from the response at $3 / 4$ of the span.

The answer to all three questions is given within a tolerance of $5 \%$, i.e., if the maximum response is not obtained at mid-span but the response obtained at mid-span is $95 \%$ of the maximum or greater, an affirmative answer is given to question (1). Similar treatment is given to questions (2) and (3).

The results of the analysis allow to extract the following conclusions:

1. At speed lower than $V_{\max }\left(\Lambda<\Lambda_{\text {max }}\right)$ the maximum displacement is obtained at mid-span for all 16 values of the $L / d$ ratio. Therefore, in all cases the answer to question (1) is affirmative with regard to displacements.

2. On the contrary, there are five values of $L / d$ such that the maximum acceleration at speed lower than $V_{\max }$ is not obtained at mid-span. These values are $1.5,1.75,2.25,2.5$, and 3.75 . In all these cases the maximum acceleration is computed accurately at both sections $x / L=\frac{1}{4}$ and $x / L=\frac{3}{4}$, and therefore question (2) is answered affirmatively. A more detailed analysis of these five situations reveal the following facts: (1) For $L / d=1.5$ and 2.5 cancellation of the first resonance of the first mode occurs and the third resonance of the second mode $(\Lambda=4 / 3)$ becomes prevalent (see the next section for a more detailed explanation of cancellations). Respectively, the maximum acceleration is approximately 15 and $11 \%$ greater than the maximum value obtained at midspan. Fig. 4 shows the response corresponding to $L / d=1.5$.

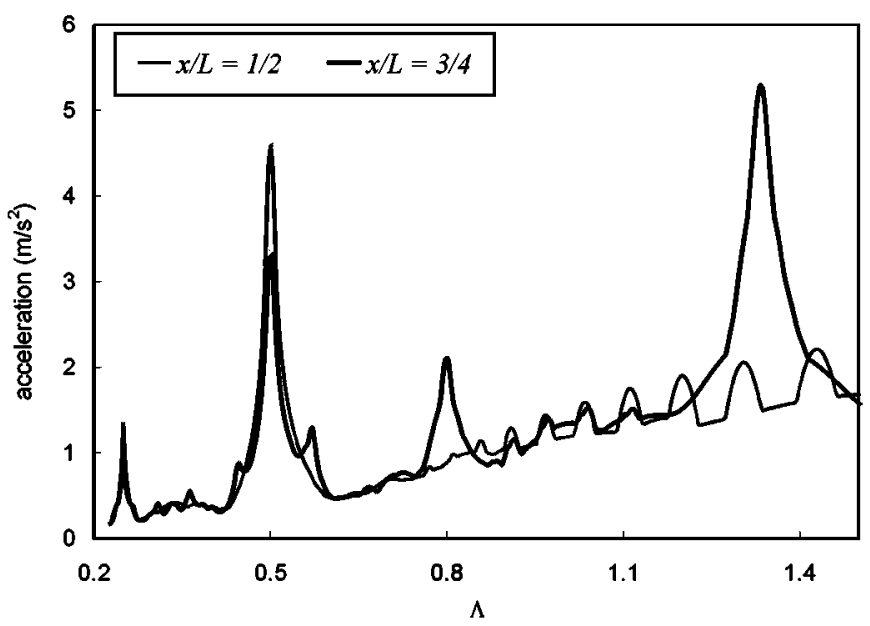

Fig. 4. Maximum acceleration at $x / L=\frac{1}{2}$ and $x / L=\frac{3}{4}$ for $L / d=1.5$

(2) For $L / d=1.75,2.25$, and 3.75 the second resonance of the second mode $(\Lambda=2)$ is predominant notwithstanding that there is no cancellation of the first resonance of the first mode. In these three cases the maximum acceleration outweighs the maximum value computed at mid-span by 11,6 , and $6 \%$, respectively. Fig. 5 shows the maximum accelerations for $L / d=2.25$. One example of this behavior would be that of a bridge of length $L=36 \mathrm{~m}$ and fundamental frequency $n_{0}=3.5 \mathrm{~Hz}$ traversed by a regular train with characteristic distance $d=16 \mathrm{~m}$. For these bridges the second resonance of the second mode is attained at $V_{2,2}=2 \cdot 3.5 \cdot 16$ $=112 \mathrm{~m} / \mathrm{s}$. (3) During the development of the $\mathrm{PhD}$ thesis (Museros 2002), the authors also found real cases such that the maximum acceleration at the first and third quarters of the span was greater than the acceleration at mid-span. One of such cases is shown in Fig. 6. The plot corresponds to the maximum acceleration at mid-span and at $3 / 4$ of the span for a bridge with the following characteristics: $L=18.5 \mathrm{~m}, m$ $=18,500 \mathrm{~kg} / \mathrm{m}, n_{0}=4.32 \mathrm{~Hz}$. The bridge is traversed by a Talgo train. The characteristics that define this type of train are available from the works of the ERRI (ERRI D-214 2001) and can also be downloaded as an ASCII text file from http://estruct7.ugr.es/ pmuseros. As can be seen, the peak acceleration is obtained at $3 / 4$ of span at

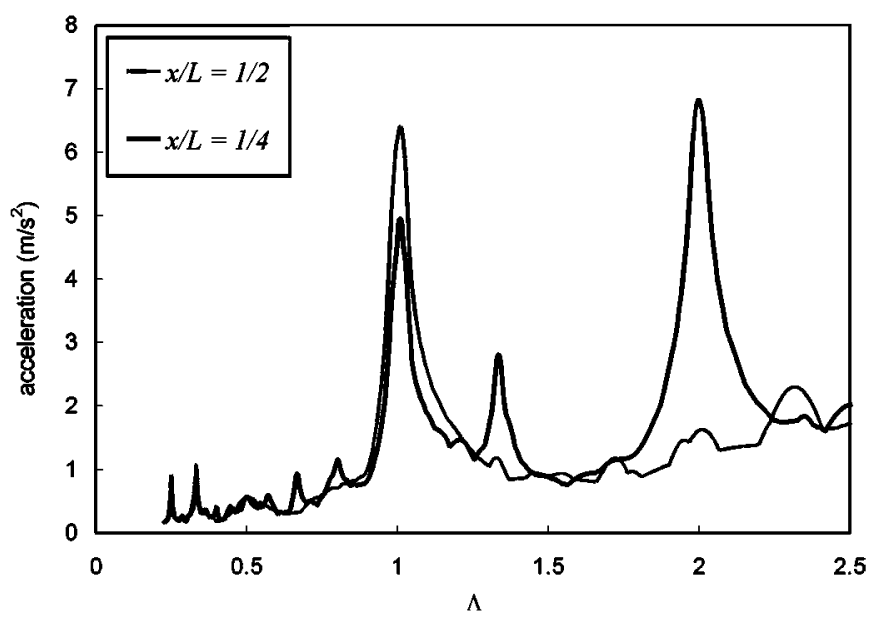

Fig. 5. Maximum acceleration at $x / L=\frac{1}{2}$ and $x / L=\frac{1}{4}$ for $L / d=2.25$ 


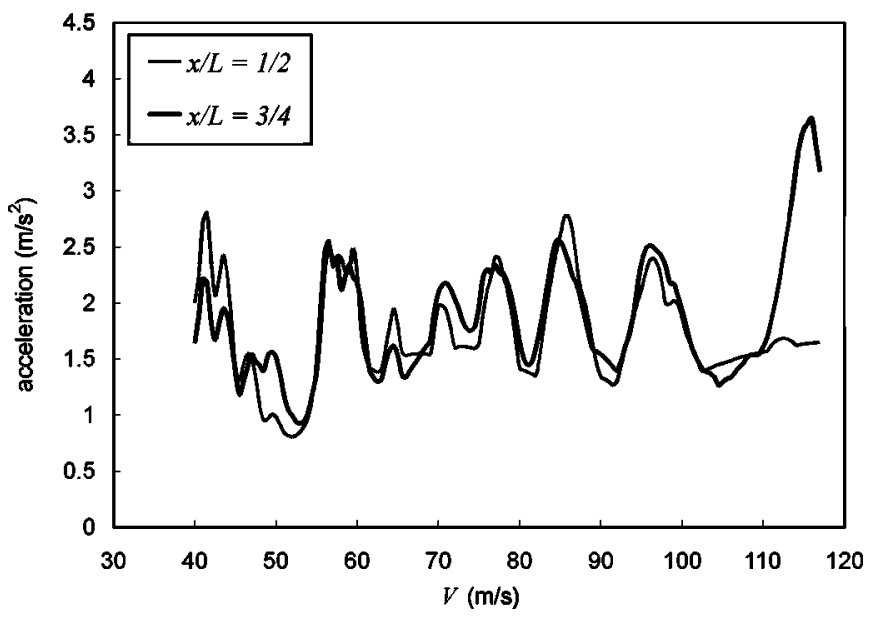

Fig. 6. Maximum acceleration at $x / L=\frac{1}{2}$ and $x / L=\frac{3}{4}$ in bridge of $L$ $=18.5$ and $n_{0}=4.32 \mathrm{~Hz}$ traversed by the Talgo high-speed train

$115.83 \mathrm{~m} / \mathrm{s}(417 \mathrm{~km} / \mathrm{h})$. The peak value is $3.64 \mathrm{~m} / \mathrm{s}^{2}$, while the maximum mid-span acceleration is $2.8 \mathrm{~m} / \mathrm{s}^{2}$, i.e., $30 \%$ less. Though not shown in the figure, the acceleration at the first quarter of the span is only slightly lower than at 3/4 of the span. It should be emphasized that, if the maximum speed at the site is fixed at $97.22 \mathrm{~m} / \mathrm{s}(350 \mathrm{~km} / \mathrm{h})$, the maximum design speed according to Eurocode 1 (CEN 2002a) is $V_{\max }=1.2 \times 97.22=116.67 \mathrm{~m} / \mathrm{s}$, and thus the peak acceleration at $115.83 \mathrm{~m} / \mathrm{s}$ would be relevant for the verification of the serviceability limit states.

3. With reference to question (3), the answer is affirmative with regard to displacements and accelerations for speeds lower than $V_{\max }$ and for all 16 values of $L / d$.

4. Finally, if the limitation $V \leqslant V_{\max }$ is removed and the results are examined for $0.225 \leqslant \Lambda \leqslant 4.4$, the maximum displacement is obtained at mid-span for the 16 different values of $L / d$. On the contrary, most of the times the maximum acceleration is not obtained at mid-span. The reason for this is the large acceleration associated with the first resonance of the second mode, as shown in Fig. 7 for $L / d=3.75$.

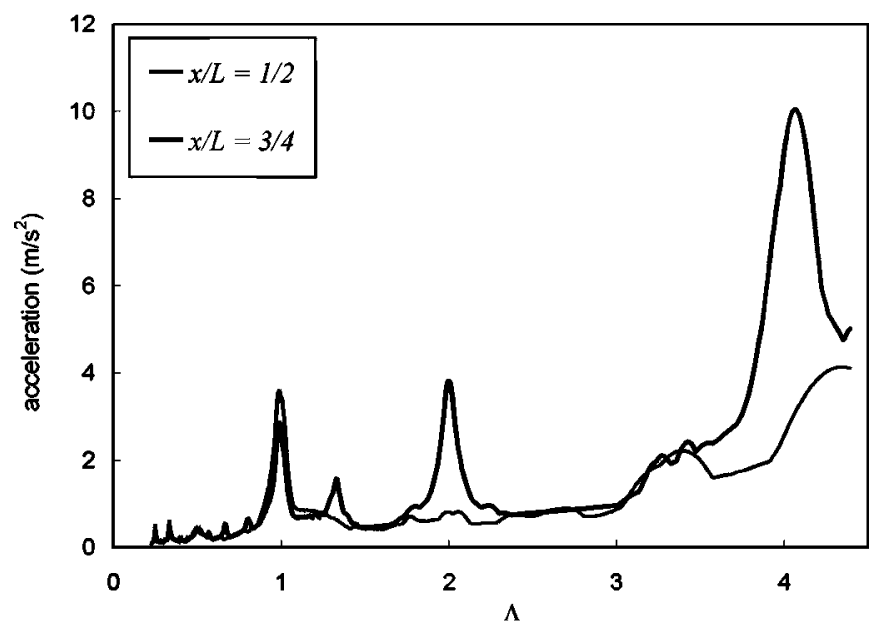

Fig. 7. Maximum acceleration at $x / L=\frac{1}{2}$ and $x / L=\frac{3}{4}$ for $L / d=3.75$

\section{Cancellation of Resonance}

Yang et al. derived the mathematical condition of cancellation for the resonances of the first mode in simply supported bridges (Yang et al. 1997), and more recently presented a study of the cancellation condition for bridges on elastic bearings (Yang et al., 2004). Restricting the discussion to the first four resonances of the fundamental mode of simply supported bridges, this condition can be expressed as

$$
\frac{L}{d}=\frac{2 j-1}{2 i} \quad i=1,2,3,4 \quad j=1,2,3, \ldots
$$

In Eq. (14) all the variables retain their usual meaning, and thus $i$ represents the resonance number; in addition, $j=$ integer number taking values equal to or greater than unity. If Eq. (14) is satisfied exactly the $i$ th resonance of the first mode will not take place even if the velocity of the train is equal to the theoretical resonance speed $V_{1, i}$. If it satisfied approximately, the resonant amplification will be kept to a low level. As explained in (Yang et al. 1997), the physical reason for this phenomenon is the suppression or cancellation of the free vibrations associated with the loads that in any given instant have abandoned the bridge; as a consequence, the oscillations do not accumulate and the behavior of the structure is governed solely by the effects of the last load.

Eq. (14) explains satisfactorily why high-speed trains cannot induce resonance situations in some railway bridges; an example of such behavior is the passage of an ICE over a $40 \mathrm{~m}$ simply supported bridge: for this train, the characteristic distance between consecutive groups of loads is equal to $26.4 \mathrm{~m}$, leading to $L / d=40 / 26.4=1.515$. This value corresponds approximately to the ratio $L / d$ predicted by Eq. (14) for $i=1$ and $j=2$.

Similarity formulas $(4 a)$ and $(4 b)$ guarantee that if cancellation of resonance of any mode occurs for a certain train-bridge system, it will also occur for all similar systems. Based on this fact, some interesting conclusions can be drawn from the calculations carried out in "Influence of Second Bending Mode on Response of Bridge." As explained in that section, a train consisting of 15 equally spaced loads has been considered and the closed-form solution of Eq. (1) has been obtained for all $L / d$ ratios contained in Table 1. The results of this analysis have furnished the values of $L / d$ such that cancellation occurs. These values have been collected in Table 2 for the resonances of the second mode. Regarding the resonances of the first mode, Eq. (14) suffices for predicting the cancellation situations. It should be emphasized that Table 2 does not contain all possible values of $L / d$ causing cancellation of resonance; instead, it contains only those values causing cancellation among the 16 different $L / d$ ratios listed in Table 1 .

\section{Conclusions}

The results of the investigation presented in this paper can be summed up in the following general conclusions:

1. The resonant behavior of simply supported bridges under the passage of trains of equally spaced loads has been analyzed. A type of train consisting of 15 equally spaced loads has been utilized in order to excite the structures at resonance and compare the relative influence of the first and second bending modes.

2. At speeds lower than $116.67 \mathrm{~m} / \mathrm{s}(420 \mathrm{~km} / \mathrm{h})$, for 16 different values of the $L / d$ ratio comprised in the interval 0.3 
Table 2. Values of $L / d$ Such That Cancellation of Resonance Occurs for Second Bending Mode

\begin{tabular}{|c|c|c|c|c|c|c|c|c|c|}
\hline$L / d$ & $i=1$ & $i=2$ & $i=3$ & $i=4$ & $L / d$ & $i=1$ & $i=2$ & $i=3$ & $i=4$ \\
\hline 0.30 & - & - & - & - & 2.25 & - & - & - & $\mathrm{X}$ \\
\hline 0.50 & - & - & - & $X$ & 2.50 & - & $X$ & - & $\mathrm{X}$ \\
\hline 0.75 & - & - & - & $\mathrm{X}$ & 2.75 & - & - & - & $X$ \\
\hline 1.00 & - & $X$ & $X$ & $X$ & 3.00 & $X$ & $X$ & $X$ & $\mathrm{X}$ \\
\hline 1.25 & - & - & - & $\mathrm{X}$ & 3.25 & - & - & - & $\mathrm{X}$ \\
\hline 1.50 & - & $X$ & - & $X$ & 3.50 & - & $X$ & - & $X$ \\
\hline 1.75 & - & - & - & $X$ & 3.75 & - & & - & $X$ \\
\hline 2.00 & $X$ & $X$ & $\mathrm{X}$ & $\mathrm{X}$ & 4.00 & $X$ & $X$ & $\mathrm{X}$ & $\mathrm{X}$ \\
\hline
\end{tabular}

$i=$ resonance number; cancellations marked with $\mathrm{X}$.

$\leqslant L / d \leqslant 4.0$, no case has been found such that the maximum displacement is not obtained at mid-span.

3. Conversely, it has been confirmed that in several cases the maximum acceleration at speeds lower than $116.67 \mathrm{~m} / \mathrm{s}$ does not correspond to the mid-span section. This is due to the development of resonance situations associated with the second bending mode, and consequently the contribution of this mode cannot be disregarded. In these cases the maximum acceleration could be computed accurately either at the first quarter of the span or at $3 / 4$ of the span since there was very little difference between the results at these two sections. It is of interest to mention that, since in many cases the maximum value at mid-span is not exceeded by a large amount, it is likely that the maximum accelerations would take place at this section providing that the damping ratio assigned to the second mode was higher than the one assigned to the first mode.

4. If the speed range is extended in order to encompass the first four resonances of the first and second mode, the maximum displacement is still obtained at mid-span for all 16 values of $L / d$. On the contrary, in only a few cases does the maximum acceleration correspond to the mid-span section: this is a consequence of the high accelerations produced by the first resonance of the second mode.

5. The maximum displacements obtained at the first quarter of the span and at $3 / 4$ of the span have been found to be very similar (difference less than 5\%) for all speeds up to the first resonance of the second mode. The same behavior has been observed for the accelerations at speeds lower than $116.67 \mathrm{~m} / \mathrm{s}$. Conversely, when speeds up to the first resonance of the second mode are considered some cases have been identified such that the maximum accelerations differ significantly (more than 5\%).

6. The results of the present investigation show no contradiction with the usual assumption that the fundamental bending mode suffices for the determination of the maximum vertical displacement, as indicated by other authors (Biggs 1964; Smith 1988; Yang et al. 1997; Li and Su 1999). On the contrary, assigning the same damping ratio to the first and second modes several cases have been identified such that the maximum acceleration is not obtained at mid-span. This implies that, in general, the contribution of the second bending mode cannot be neglected for the determination of the maximum acceleration in simply supported bridges.

7. The resonant vibration of the first and second modes is cancelled for particular values of the $L / d$ ratio. The cancellations of the first mode were analyzed previously by other authors. A number of values of $L / d$ such that cancellation occurs for the second mode have been identified in the present investigation.

\section{Acknowledgment}

The writers wish to express their gratitude to Mr. Jorge Nasarre, from the Spanish National Railways (RENFE), also a member of the ERRI D-214 Committee, for his help and encouragement during the development of this investigation.

\section{Appendix: Derivation of Dimensionless Equation of Motion}

The partial differential equation governing the behavior of the simply supported beam of Fig. 1 can be found in the works of a number of authors as, for instance, Frýba (1999). This wellknown equation is as follows:

$$
m \frac{\partial^{2} y}{\partial t^{2}}+\frac{\partial^{2}}{\partial x^{2}}\left(E I \frac{\partial^{2} y}{\partial x^{2}}\right)=q(x, t)
$$

In Eq. (15) it is assumed that the $Y$ axis is a vertical axis of symmetry of the cross section of the beam, and the shear deformation and rotary inertia of the beam are neglected. The following notation is used: $m=$ constant mass per unit length; $y=y(x, t)=$ deformed shape of the beam at time $t$; and $x=$ abscissa corresponding to a particular section; also, $E=$ modulus of elasticity and $I=$ second moment of area of the cross section (both are assumed to be constant along the span); finally, $q(x, t)$ stands for the distributed load per unit length acting on the beam at the section corresponding to $x$ and at time $t$ (according to the $Y$ axis, $q$ is positive if directed upwards).

The deformed shape of a simply supported beam is expressed as a linear combination of the family of sines $\phi_{j}(x)$ as in Eq. (3), and the load is represented by means of Dirac delta functions acting at the point $x=V t-d_{k}$, where $V$ is the speed of the train and $d_{k}$ is the distance from the $k$ th load to the beginning of the bridge

$$
q(x, t)=-\sum_{k=1}^{N P}\left(H\left(t-\frac{d_{k}}{V}\right)-H\left(t-\frac{d_{k}+L}{V}\right)\right) P_{k} \delta\left(x-\left(V t-d_{k}\right)\right)
$$

In Eq. (16) $N P=$ total number of loads; $P_{k}=$ value of the $k$ th load; $\delta\left(x-x_{0}\right)=$ Dirac delta, and $H\left(t-t_{0}\right)=$ Heaviside unit function acting at time $t_{0}$. 
If the Eqs. (3) and (16) are introduced in Eq. (15), after multiplication by the $n$th sine $\phi_{n}(x)$ and integration along the span of the beam $(L)$ the equation of motion for the $n$th mode is obtained. In addition, if a viscous damping $\zeta_{n}$ is introduced to account for the energy dissipation, the equation of motion for the $n$th mode transforms into

$$
\begin{aligned}
\ddot{\xi}_{n}(t) & +2 \xi_{n} \omega_{n} \dot{\xi}_{n}(t)+\omega_{n}^{2} \xi_{n}(t) \\
& =\frac{-2}{m L} \sum_{k=1}^{N P}\left(H\left(t-\frac{d_{k}}{V}\right)-H\left(t-\frac{d_{k}+L}{V}\right)\right) P_{k} \sin \frac{n \pi\left(V t-d_{k}\right)}{L}
\end{aligned}
$$

In Eq. (17) $\omega_{n}$ represents the frequency of the $n$th mode in radians/second

$$
\omega_{n}=\left(\frac{n \pi}{L}\right)^{2} \sqrt{\frac{E I}{m}}
$$

The fundamental frequency in Hertz $\left(n_{0}\right)$ and the fundamental period $(T)$ are computed straightforwardly from Eq. (18). Though it corresponds to the first mode, the natural frequency is denoted quite often by a subscript " 0 " : $n_{0}=\omega_{0} /(2 \pi)=\omega_{1} /(2 \pi) ; T=1 / n_{0}$. Eq. (17) can be written in dimensionless form using a dimensionless time $\tau=t / T=\omega_{0} t /(2 \pi)$. In such a case, the time derivatives of the modal amplitude $\xi_{n}$ with respect to real time (denoted with dots) and with respect to dimensionless time (denoted by primes), follow relations similar to Eq. (5).

The following relations are also useful:

$$
\begin{gathered}
H\left(t-\frac{d_{k}}{V}\right)=H\left(\tau-\frac{d_{k}}{V T}\right)=H\left(\tau-\frac{d_{k}}{\alpha L}\right) \\
H\left(t-\frac{d_{k}+L}{V}\right)=H\left(\tau-\frac{d_{k}+L}{V T}\right)=H\left(\tau-\frac{d_{k}}{\alpha L}-\frac{1}{\alpha}\right) \\
\frac{n \pi\left(V t-d_{k}\right)}{L}=n \pi\left(\alpha \tau-\frac{d_{k}}{L}\right) \quad \omega_{n}^{2}=n^{2} \omega_{0}
\end{gathered}
$$

Finally, substituting Eqs. (19) into Eq. (17), and taking into account the definition of dimensionless time and the relations between the real and dimensionless time derivatives, the equation of motion (1) is obtained immediately.

\section{Notation}

The following symbols are used in this paper:

$a=$ vertical acceleration of section of bridge;

$d=$ distance between consecutive loads, or distance from load to origin of train;

$E=$ modulus of elasticity of bridge;

$H=$ Heaviside unit step function;

$I=$ second moment of area of cross section of bridge;

$i=$ resonance number;

$j=$ positive integer index;

$L=$ span length;

$L_{\max }=$ maximum length associated to particular value of $L / d$ ratio;

$L_{\min }=$ minimum length associated to particular value of $L / d$ ratio;

$m=$ mass per unit length;
$N=$ number of bending modes considered in series expansion;

$N P=$ number of loads of train;

$n=$ integer indicating $n$th bending mode;

$n_{0}=$ fundamental frequency (Hz) (first bending mode);

$P=$ value of axle load;

$q=$ distributed load acting on bridge;

$T, T_{1}=$ fundamental period (first bending mode);

$t=$ real time;

$V=$ speed of train;

$V_{\text {max }}=116.67 \mathrm{~m} / \mathrm{s}(420 \mathrm{~km} / \mathrm{h})$;

$V_{n, i}=$ speed of train corresponding to $i$ th resonance of $n$th bending mode;

$x=$ abscissa:

$y=$ vertical displacement of section of bridge;

$\alpha=$ dimensionless speed;

$\alpha_{\max }=$ dimensionless speed corresponding to $V_{\max }$;

$\alpha_{n, i}=$ dimensionless speed corresponding to $i$ th resonance of $n$th bending mode;

$\delta=$ Dirac delta function;

$\zeta=$ damping ratio;

$\Lambda=$ normalized wavelength;

$\Lambda_{\max }=$ normalized wavelength corresponding to $V_{\max }$;

$\Lambda_{n, i}=$ normalized wavelength corresponding to $i$ th resonance of $n$th bending mode;

$\xi=$ amplitude of bending mode;

$\tau=$ dimensionless time;

$\tau_{0}=$ particular value of dimensionless time;

$\phi=$ bending mode;

$\omega=$ frequency of bending mode $(\mathrm{rad} / \mathrm{s})$; and

$\omega_{0}, \omega_{1}=$ fundamental frequency $(\mathrm{rad} / \mathrm{s})$ (first bending mode).

\section{Subscripts}

$$
\begin{aligned}
k= & \text { values corresponding to } k \text { th load; } \\
n= & \text { values corresponding to } n \text {th bending mode; and } \\
1,2= & \text { values corresponding to two different train- } \\
& \text { bridge systems. }
\end{aligned}
$$

Biggs, J. M. (1964). Introduction to structural dynamics, McGraw-Hill, New York.

European Committee for Standardization (CEN. (2002a). "Actions on structures. Part 2: General actions-Traffic loads on bridges. Final Draft prEN 1991-2," Eurocode 1, Brussels, Belgium.

European Committee for Standardization (CEN. (2002b). "Basis of Structural Design. Annex A2: Application for bridges. Final PT Draft EN 1990-prAnnex A2." Brussels, Belgium.

European Rail Research Institute, D-214 Committee (ERRI D-214. (2001). "Ponts-Rails pour vitesses $>200 \mathrm{~km} / \mathrm{h}$. Rapport Final." Rep. No. 9, Utretch, France (in French).

Frýba, L. (1996). Dynamics of railway bridges, Thomas Telford, London. Frýba, L. (1999). Dynamics of solids and structures under moving loads, 3rd Ed., Thomas Telford, London.

Frýba, L. (2001). "A rough assessment of railway bridges for high speed trains." Eng. Struct., 23, 548-556.

Klasztorny, M., and Langer, J. (1990). "Dynamic response of single-span beam bridges to a series of moving loads." Earthquake Eng. Struct. Dyn., 19, 1107-1124.

Li, J., and Su, M. (1999). "The resonant vibration for a simply supported girder bridge under high-speed trains." J. Sound Vib., 224(5), 897- 
Museros, P. (2002). "Vehicle-bridge interaction and resonance effects in simply supported bridges for high speed lines." $\mathrm{PhD}$ thesis, Superior School of Industrial Engineering, Technical Univ. of Madrid, Madrid, Spain (in Spanish).

Museros, P., and Alarcón, E. (2002). "An investigation on the importance of train-bridge interaction at resonance." Proc., 6th Int. Conf. on Computational Structures Technology, Civil-Comp Press, Stirling, Scotland, 335-336.

Museros, P., Romero, M., Poy, A., and Alarcón, E. (2002). "Advances in the analysis of short span railway bridges for high speed lines." Comput. Struct., 80(27-30), 2121-2132.

Museros, P., Vivero, G., and Alarcón, E. (1999). "Moving loads on rail- way bridges: the Spanish Code approach." Proc., 4th European Conf. on Structural Dynamics (Eurodyn '99), A.A. Balkema, Rotterdam, The Netherlands, 675-680.

Olsson, M. (1991). "On the fundamental moving load problem." J. Sound Vib., 145(2), 299-307.

Smith, J. W. (1988). Vibration of structures: Applications in civil engineering design, Chapman \& Hall, London.

Yang, Y. B., Lin, C. L., Yau, J. D., and Chang, D. W. (2004). "Mechanism of resonance and cancellation for train-induced vibrations on bridges with elastic bearings." J. Sound Vib., 269(1-2), 345-360.

Yang, Y. B., Yau, J. D., and Hsu, L. C. (1997). "Vibration of simple beams due to trains moving at high speeds." Eng. Struct., 19(11), 936-944. 